\title{
A low cost reconfigurable mini-array facility for (under)graduate studies in cosmic ray physics
}

\author{
Chiara Pinto*1,2, Paola La Rocca ${ }^{1,2}$, Davide Nicotra ${ }^{3}$, Orazio Parasole ${ }^{1,2}$ and \\ Francesco Riggi ${ }^{1,2}$
}

1. Department of Physics and Astronomy E.Majorana, University of Catania, Via S. Sofia 64, Catania, Italy

2. INFN, Sezione di Catania, Via S. Sofia 64, Catania, Italy

3. Centro Siciliano di Fisica Nucleare e Struttura della Materia, Via S. Sofia 64, Catania, Italy

E-mail: chiara.pinto@ct.infn.it

\begin{abstract}
Coincidence measurements between cosmic ray detectors placed some distance apart are the standard way to detect extensive air showers created in the Earth atmosphere. While the detection of the highest energy cosmic rays requires distances in the order of $\mathrm{km}$, compact arrays would probe the low energy region of the energy spectrum of primary particles. In this Project we exploited the potential of a detection strategy based on the use of small area individual detectors, which can be easily moved and re-configured in different detection geometries, to be employed for different physics investigations. An important aspect of this Project is the educational activity in cosmic ray physics being planned for undergraduate and graduate students, together with the outreach and citizen science involvements. However, also a series of physics investigations (among which the study of muon bundles and of nearly horizontal showers) are within the reach of this facility, when operated in stand-alone mode or in combination with other existing detectors. In order to keep as low as possible the overall cost of the facility, the layout of each detection module is based on scintillator tiles, Wavelength Shifter (WLS) bars and Silicon Photomultipliers (SiPM) for light collection and readout. Signals from each detector are discriminated and shaped, then sent to an Arduino MEGA board for triggering, data acquisition, GPS time stamping and event storage. A first set of 30 detection modules were already fully built and characterized. Commissioning measurements under different topological configurations of the array have been carried out both in stand-alone mode and in coincidence with other cosmic ray detectors.
\end{abstract}

European Physical Society Conference on High Energy Physics - EPS-HEP2019 -

10-17 July, 2019

Ghent, Belgium

${ }^{*}$ Speaker. 


\section{Educational and Outreach activities with cosmic ray physics in Catania}

Educational experiments with cosmic rays provide a great opportunity to learn more about physics in many fields. There are many examples of collaborations which involve high schools, university and research centres in projects concerning cosmic ray physics. These activities offer to school teachers and associated groups of students the possibility to be trained in the world of experimental high energy physics, since not only may they learn the basics of cosmic ray physics, but also stay in touch with several aspects of a real research activity [1]. Cosmic rays are intimately connected to other branches of physics. First of all, particle and nuclear physics are essential fields of research strictly linked to cosmic rays, since many of the particles which constitute the nuclear world were first observed within the cosmic radiation. The interactions of such particles with nuclei, as they happen in the Earth's atmosphere, are an important ingredient in the understanding of the particle properties and classification of the fundamental interactions. Moreover, important progresses in the development of detection techniques and devices which are now the basic tools in nuclear and particle physics were made in the course of investigations concerned with cosmic ray physics. Astrophysics, cosmology, space physics, solar physics and physics of the atmosphere are just a few additional examples of fields where the connection with cosmic ray physics plays a major role. Due to the many facets of cosmic ray physics, and the possible interplay with so many disciplines, a high impact on science education is expected, and has actually been observed from the very beginning. Cosmic ray physics provides indeed good opportunities for educational activities and experiments, not so easy to envisage in traditional nuclear and particle physics.

Our group in Catania is involved since many years in several activities concerning cosmic rays physics, from local initiatives to the participation to a large international Collaboration (The EEE Project, [2]). An important aspect of our work is aimed to the educational impact of our project, which is planned for undergraduate and graduate students in cosmic ray physics, together with the outreach and citizen science involvements during public town events such as Researchers Night or the Open Labs.

\section{The mini-array project}

The proposed project is organized as an easily reconfigurable mini-array of detectors made by small size individual detection modules. In the present stage of the project a simple digital readout of the hit modules has been employed, which allows for a reconstruction of the geometrical pattern of each detected event with its occurrence probability, complemented by a time tagging of the event obtained by the GPS time information. For this task, low cost solutions were exploited both for the detectors and front-end electronics, as well as for the trigger, time-stamping of the events and data acquisition and storage. The basic elements of the setup are described in the following. As shown in Fig. 1, for each detection module, these basic components were installed in a light-tight black box (approximate size $40 \times 30 \times 13.5 \mathrm{~cm}^{3}$ ): the scintillator tile and the WLS bar wrapped with aluminized tape, the Silicon Photomultiplier, a discriminator/shaper board and a power supply board providing the $\pm 5 \mathrm{~V}$ to the amplifier and discriminator boards. A BNC connector provides the output TTL shaped signal to the data acquisition and trigger system, while banana connectors are used to provide the bias voltage to the photosensor. 


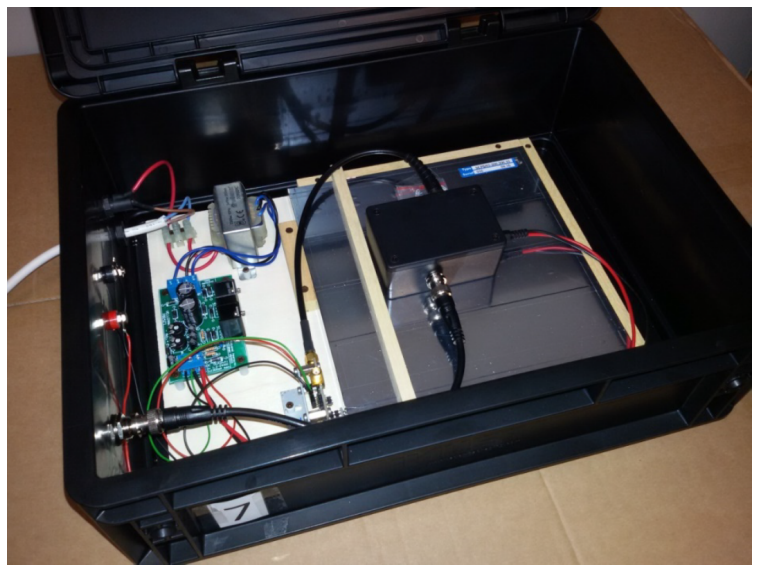

Figure 1: Picture of one of the 30 detection modules of the mini-array. The boxes have been homeassembled in the Physics Department of Catania University.

\subsection{Detectors and photosensors}

Each detection module makes use of a small size $\left(20 \times 20 \times 1 \mathrm{~cm}^{3}\right)$ extruded scintillator tile coupled to a Wavelength Shifter (WLS) bar $\left(20 \times 1 \times 1 \mathrm{~cm}^{3}\right)$ through one of its lateral sides. As reported in a previous paper [3], fully GEANT4 simulations were carried out with the aim of evaluating the light collection efficiency under various detection configurations and study the influence of different key factors on the average number of collected photons per event. The individual efficiency of each module was measured with respect to a three-fold coincidence trigger given by other detectors of the same size assembled in a telescope configuration. A first set of 9 detection modules of the array were equipped with Silicon photomultipliers devices, with a sensitive area of $4 \times 4 \mathrm{~mm}^{2}$, a peak efficiency (PDE 32.5\%) at $550 \mathrm{~nm}$ and a breakdown voltage around 28.7 V. Due to the results of previous tests [4], all subsequent 21 modules were built with larger area and low dark current SiPMs, employing the $6 \times 6 \mathrm{~mm}^{2}$ Hamamatsu models, with a breakdown voltage around 38.7 V. These allowed to reach a detection efficiency close to $100 \%$. Fig. 2 shows the individual detection efficiency for all the modules. Bias voltage for the SiPMs was provided to the modules by two independent external power supplies. Both circuits made use of a temperature controlled bias adjustment, based on the use of a negative temperature coefficient (NTC) thermistor.

\subsection{Electronics and data acquisition}

The signal from each SiPM is sent to a trans-impedance fast amplifier. After amplification, the signal may be sent either directly outside the detector box (for check, oscilloscope visualization or use of external standard electronics), or to a discriminator/shaper, placed inside the box, and from that to the outside. A solution based on a low-cost Arduino board was exploited, trying to push the performance of this system to its limits, still maintaining at a reasonable level the complexity of the setup. Shaped TTL signals from each detection module were sent to the digital inputs of an Arduino MEGA board through a "concentration box", which may handle up to 50 inputs. In order to treat a large number of channels in coincidence, fast digital readout instructions were implemented. As a result, a time of the order of $800 \mathrm{~ns}$ per channel was obtained, which allows to read out 30 channels - as in our measurements - in less than $30 \mu \mathrm{s}$. In the sequence of Arduino digital channels, some 


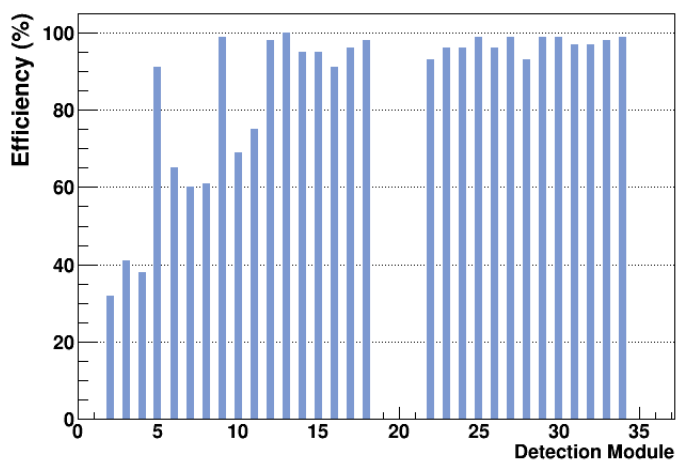

Figure 2: Individual detection efficiency of the 30 modules employed in the present work, as measured by the ratio between four-fold and three-fold coincidences in a telescope configuration. Some of the first modules built were equipped with $4 \times 4 \mathrm{~mm}^{2}$ SiPMs, exhibiting detection efficiencies between $35 \%$ and $70 \%$, while all the remaining modules made use of $6 \times 6 \mathrm{~mm}^{2}$, low dark current devices, reaching efficiencies close to $100 \%$.

of them are dedicated to specialized operations: for instance the communication to the PC, the handling of the 1 PPS GPS signal and the connection to pressure and temperature sensor. After the fast readout of all channels, a time stamp of the event is obtained through the information provided to Arduino by an external GPS engine, and the channel multiplicity $m$ (i.e. the number of channels which presented a low-to-high transition in each event) is built. A software trigger may then be defined, which allows to validate and store the event on the basis of the channel multiplicity (for instance triggering on events with $m>2$, i.e. at least three-fold coincidences) or selecting specific patterns of detection configurations.

\subsection{GPS time stamping of events}

For the purpose of identifying the arrival time of the events of interest and correlate them to those measured from other detectors, we also provided a GPS time stamping to each detected event, making use of a GPS module and of the clock of the Arduino board. A GPS module providing the UTC time in NMEA format and the 1 PPS (Pulse Per Second) signal was used to increase the long term stability of the Arduino's clock, with an external antenna which was usually attached to one of the external walls of the building. The number of GPS satellites as seen by the antenna was checked every 30' and in most of the cases (>90\%) this number was larger than 5.

\section{Preliminary measurements with the mini-array}

\subsection{Running conditions}

The measurements were carried out from April 19, 2019 to May 2, 2019, for a total of about 13 days, in the Lecture Hall of the Physics Department of Catania University. Thirty operating modules were employed throughout all the measurement, with a fixed geometrical configuration, as represented in Fig. 3. Temperature and atmospheric pressure were measured in small time steps (30 minutes) along all the 13 days period, in order to investigate the dependence of individual and 
coincidence rates on these parameters. The acquisition trigger for such measurements was given by at least a three-fold coincidence between any detection module, in order to suppress most of the spurious rate. Actually, three-fold coincidences are still overestimated by about $25 \%$ due to the contribution from spurious counting, whereas for $m>3$ such contribution is negligible.

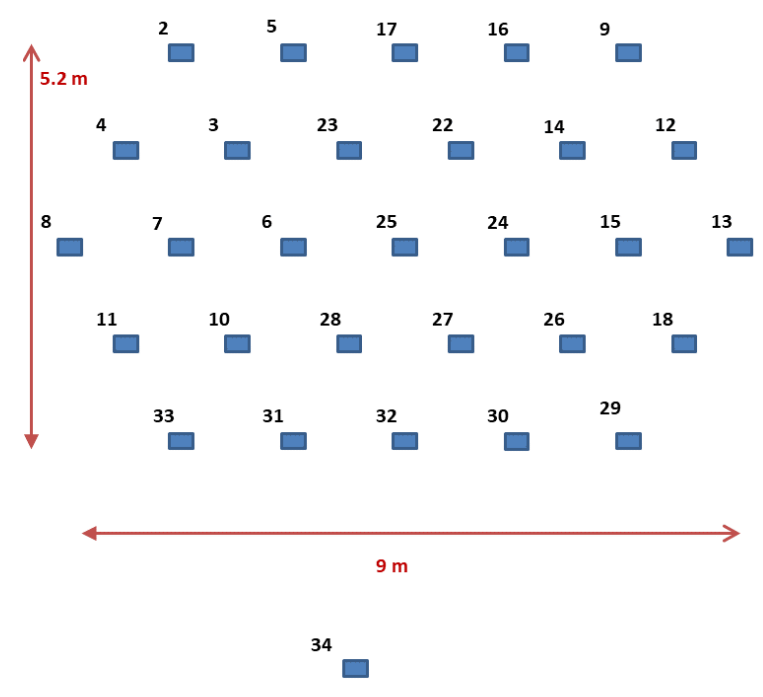

Figure 3: Geometrical configuration for commissioning tests: 30 modules distributed over an area of roughly $50 \mathrm{~m}^{2}$. An hexagonal configuration is used for all the modules (except for module 34), with a side of the triangles equal to $1.5 \mathrm{~m}$.

\subsection{Multiplicity distributions and coincidence measurements}

The coincidence between different modules in the array allows to detect the arrival of an extensive air shower. The raw multiplicity distribution of the collected events in this run is shown in the left panel of Fig.4. Coincidences involving even a large number of modules, up to the maximum allowed multiplicity, were observed. The raw multiplicity is related to the average shower particle density over the detection area. Taking into account the integrated sensitive area of all detection modules $\left(1.2 \mathrm{~m}^{2}\right.$ ) and the area where such modules were deployed (about $50 \mathrm{~m}^{2}$ ), average particle densities between $2.5 \mathrm{~m}^{-2}$ and $25 \mathrm{~m}^{-2}$ may be roughly estimated.

The availability of the GPS time stamping of events allows to study off-line coincidences with other detectors. During the commissioning tests, for about 1.5 days data were collected using an additional detector (also equipped with a GPS module) placed at about $20 \mathrm{~m}$ distance from the mini-array. The inclusive raw multiplicity distribution measured by the array was then compared to that obtained from the coincidence measurement with the additional detector (as shown in the right panel of Fig. 4), selecting a time window around the coincidence peak of about $\pm 15 \mu \mathrm{s}$. The average detected multiplicity increased from a value of 3.6 (inclusive distribution) to 6.5 (coincidence distribution), reflecting the detection of showers with a higher local particle density. 

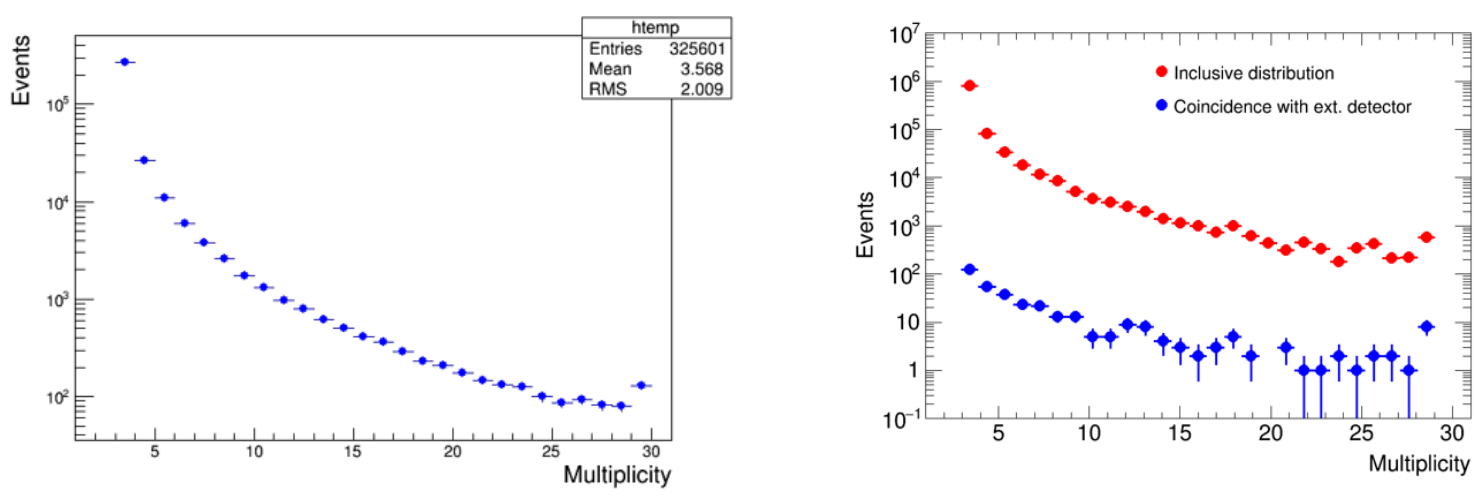

Figure 4: Left : Raw multiplicity distribution of the events collected by the mini-array during the commissioning tests. Right : Comparison between the inclusive (red points) and coincidence (blue points) multiplicity distributions measured by the array in stand-alone mode and for coincidence events with an additional muon detector placed about $20 \mathrm{~m}$ apart.

\section{Conclusions}

A small array of cosmic ray detectors has been built with a main emphasis on graduate teaching activities in a cosmic ray laboratory course addressed to master and $\mathrm{PhD}$ students in Physics. The overall cost of the facility was kept as low as possible using scintillator tiles coupled to WLS bars and SiPMs for light collection and readout. A cheap solution was found also for triggering and data acquisition, making use of an Arduino MEGA board. The apparatus was also equipped with a GPS module in order to have a time stamping of each event. Commissioning measurements using the first set of 30 detection modules available were carried out and presented in this talk.

The technology employed for the mini-array makes possible to introduce students to the main aspects of building a cosmic ray detector based on scintillators. During the construction and testing of modules, the commissioning tests, the data taking and analysis, the involvement of students was at all levels, from high school to university graduate. The main features of the array, namely its modularity and the easy reconfigurability make the mini-array a well suited apparatus for further studies of extensive air showers at different altitudes. This apparatus can also allow the study of inclined showers and the measurement of the decoherence curve, besides many other topics of interest in cosmic ray physics. The work already carried out and several possible improvements of this project suggest that this is a good possibility to learn about detection techniques, electronics and data acquisition, simulation procedures, data analysis and comparison to theoretical calculations, which highlights once more the great educational impact of such a project.

\section{References}

[1] F. Blanco, P. La Rocca and F. Riggi, Educational Experiments with Cosmic Rays, in Science in Focus, M.V. Thomase Ed., Nova Publishers, New York, U.S.A., (2008), 127-172, ISBN 1-60021-949-7.

[2] EEE collaboration website: https://eee.centrofermi.it/

[3] P.La Rocca et al., JINST 13(2018)P10013

[4] P.La Rocca et al., JINST 14(2019)T01003 